\title{
DISCUSSIONS
}

\section{The double sliding, free rotating model for granular assemblies}

\author{
DE JOSSELIN DE JONG, G. (1971). Géotechnique 21, No. 2, 155-163.
}

\section{R. J. Evans, University of Washington, and R. E. Gibson, King's College, University of London}

The Author is to be congratulated on his novel approach to this difficult subject. There are, however, two main points in the Note which we have difficulty in accepting.

First, inequalities (22) are certainly sufficient but surely not necessary to satisfy thermodynamic requirements. From (21) it is true that the condition

$$
(a+b) \geqslant 0
$$

is necessary for positive energy dissipation, but it is also sufficient and it does not seem necessary that $a$ and $b$ must separately be greater than zero; any combination satisfying the condition $(a+b) \geqslant 0$ is acceptable.

Second, inequality (23) which results from (22) is surely not invariant under co-ordinate rotation. It can certainly be shown that

$$
\left(V_{x, x} \geqslant-V_{y, y}\right) \cos 2 \psi+\left(V_{x, y}+V_{y, x}\right) \sin 2 \psi \geqslant 0
$$

which corresponds to (i) is invariant, but we believe that the inequality including the middle term of (23) is not invariant.

\section{Author's reply to R. J. Evans and K. E. Gibson}

I am grateful to Evans and Gibson for having raised these two points because my presentation of the double sliding, free rotating model was not satisfactory with respect to the first point and too short to cover the second. Both points, however, do not invalidate the essential result (the consecutive inequality (23)), which remains adequate in its presented form.

With respect to the first point I agree that the requirement of positive total energy dissipation only provides

$$
(a+b) \geqslant 0
$$

and this does not imply the more stringent condition (22), which is

$$
\begin{aligned}
& a \geqslant 0 \\
& b \geqslant 0
\end{aligned}
$$

In the Note, instead of treating the total energy, I should have pointed out that the frictional character of the sliding mechanism guarantees that every little bit of energy, wherever dissipated, is positive. The thermodynamic requirement of positive energy dissipation can therefore be stated in the more special form that the energy dissipated by each sliding mechanism separately is positive. Therefore, the reasoning beginning on the fourth line from the bottom of p. 160, up to equation (22) would have been more appropriate, if it had been presented in the following manner. 
In the model the energy is only dissipated in the planes separating the sliding elements. These planes coincide with the stress characteristics and therefore the shear stress on those planes is $p \sin \phi \cos \phi$. Only the shear stress contributes to the energy dissipation, because the elements are supposed to slide without volume change, so that the normal stress cannot produce work.

The energy $E_{a}$ dissipated per unit volume by slidings of magnitude $a$ in the $S_{1}$ direction is equal to

$$
E_{a}=p a \sin \phi \cos \phi
$$

The energy $E_{b}$ dissipated per unit volume by slidings of magnitude $b$ in the $S_{2}$ direction is equal to

$$
E_{b}=p b \sin \phi \cos \phi
$$

The thermodynamic requirement applied to each part of the energy dissipation separately gives

$$
\begin{aligned}
& E_{a} \geqslant 0 \\
& E_{b} \geqslant 0
\end{aligned}
$$

which because $p \geqslant 0$ and $\phi<\frac{1}{2} \pi$ reduces to

$$
\left.\begin{array}{l}
a \geqslant 0 \\
b \geqslant 0
\end{array}\right\}
$$

These inequalities (22) are therefore necessary to guarantee that energy is dissipated in every plane of sliding separately.

Returning now to Evans and Gibson's second objection, it must be remarked that the invariance of expression (23) was only claimed for co-ordinate transformations consisting of rotation and for reflexion of two-dimensional, rectilinear orthogonal co-ordinate systems. The components of the Jacobi matrix for a transformation from the $(x, y)$ co-ordinates to the $(\xi, \eta)$ co-ordinates are in those cases;

for a rotation through an angle $\omega$

$$
\partial \xi / \partial x=\cos \omega ; \quad \partial \xi / \partial y=\sin \omega ; \quad \partial \eta / \partial x=-\sin \omega ; \quad \partial \eta / \partial y=\cos \omega ;
$$

and for a reflexion in a line at an angle $\frac{1}{2} \theta$ to the $x$-axis

$$
\partial \xi / \partial x=\cos \theta ; \quad \partial \xi / \partial y=\sin \theta ; \quad \partial \eta / \partial x=\sin \theta ; \quad \partial \eta / \partial y=-\cos \theta
$$

The Jacobian $J$ (i.e. the determinant of the Jacobi matrix (Bickley \& Gibson, 1962, p. 59) of those transformations is +1 for a rotation, and -1 for a rotation combined with a reflexion, as can be seen from the foregoing relations.

After multiplying (23) through by $p$, its middle term becomes

$$
M=-\left(V_{x, x}-V_{y, y}\right) \tau_{x y}+\frac{1}{2}\left(V_{x, y}+V_{y, x}\right)\left(\sigma_{x}-\sigma_{y}\right)
$$

which can be written in index notation as

$$
M=-\frac{1}{2} \epsilon_{i j}\left(V_{i, k}+V_{k, i}\right) \sigma_{j k}
$$

Because only rectilinear, orthogonal co-ordinates are considered no distinction need to be made between covariant and contravariant indices. The symbol $\epsilon_{i j}$ stands for the two-dimensional permutation tensor which is defined to be zero if both indices are equal, +1 if the indices form an even permutation, -1 for odd permutations. In this case its components are

$$
\begin{array}{ll}
\epsilon_{11}=\epsilon_{x x}=0 & \epsilon_{12}=\epsilon_{x y}=+1 \\
\epsilon_{21}=\epsilon_{y x}=-1 & \epsilon_{22}=\epsilon_{y y}=0
\end{array}
$$


Transition to a Greek co-ordinate system $\xi^{\alpha}$ shows that $\epsilon_{i j}$ is a weighed tensor, because

$$
\epsilon_{\alpha \beta} \frac{\partial \xi^{\alpha}}{\partial x^{i}} \frac{\partial \xi^{\beta}}{\partial x^{j}}=\frac{\partial \xi^{\alpha}}{\partial x} \frac{\partial \xi^{\beta}}{\partial y}-\frac{\partial \xi^{\alpha}}{\partial y} \frac{\partial \xi^{\beta}}{\partial x}=J . \epsilon_{i j}
$$

Transformation of the components of the velocity gradient is given by

$$
V_{i, k}=V_{\lambda, \gamma} \frac{\partial \xi^{\lambda}}{\partial x^{i}} \frac{\partial \xi^{\gamma}}{\partial x_{k}}
$$

and for the stresses

$$
\sigma_{j k}=\sigma_{\mu \nu} \frac{\partial \xi^{\mu}}{\partial x^{j}} \frac{\partial \xi^{v}}{\partial x^{k}}
$$

By transition from Roman co-ordinates to Greek co-ordinates the foregoing expression for $M$ is transformed as follows

$$
\begin{aligned}
M & =-\frac{1}{2} \epsilon_{t,}\left(V_{i, k}+V_{k, \gamma}\right) \sigma_{j k} \\
& =-\frac{1}{2} J^{-1} \epsilon_{\alpha \beta} \frac{\partial \xi^{\alpha}}{\partial x^{i}} \frac{\partial \xi^{\beta}}{\partial x^{j}}\left(V_{\lambda, \gamma}+V_{\gamma, \lambda}\right) \frac{\partial \xi^{\lambda}}{\partial x^{i}} \frac{\partial \xi^{\gamma}}{\partial x^{l \epsilon}} \sigma_{\mu \nu} \frac{\partial \xi^{\mu}}{\partial x^{j}} \frac{\partial \xi^{\nu}}{\partial x^{k}} \\
& =-\frac{1}{2} J^{-1} \epsilon_{\alpha \beta}\left(V_{\lambda, \gamma}+V_{\gamma, \lambda}\right) \sigma_{\mu v}\left(\frac{\partial \xi^{\alpha}}{\partial x^{i}} \frac{\partial \xi^{\lambda}}{\partial x^{i}}\right)\left(\frac{\partial \xi^{\beta}}{\partial x^{j}} \frac{\partial \xi^{\mu}}{\partial x^{j}}\right)\left(\frac{\partial \xi^{\gamma}}{\partial x^{k}} \frac{\partial \xi^{\nu}}{\partial x^{k}}\right) \\
& =-\frac{1}{2} J^{-1} \epsilon_{\alpha \beta}\left(V_{\lambda, \gamma}+V_{\gamma, \lambda}\right) \sigma_{\mu \nu} \delta^{\alpha \lambda} \delta^{\beta \mu} \delta^{\nu v} \\
& =-\frac{1}{2} J^{-1} \epsilon_{\alpha \beta}\left(V_{\alpha, \gamma}+V_{\gamma, \alpha}\right) \sigma_{\beta \gamma}
\end{aligned}
$$

The expressions for $M$ in Roman and Greek co-ordinates are similar, except for the presence of the factor $J^{-1}$.

For rotation $J$ is equal to +1 , and complete identity is obtained showing invariance of the expression for $M$ in that case.

For reflexion $J$ is equal to -1 and $M$ changes sign. This sign inversion does not spoil the invariance, because an introduction of equations (12) and (14) shows that

$$
M=(-a+b) \sin \phi \cos \phi
$$

Sign inversion amounts to the interchange of the shear strain rates $a$ and $b$, which correspond to the $S_{1}$ and $S_{2}$ characteristic directions respectively. This interchange is a consequence of the reflexion which corresponds to the transition from a right-handed co-ordinate system to a left-handed one.

The original inequalities expressed by (12) and (14) were

$$
-(a+b) \sin \phi \cos \phi \leqslant(-a+b) \sin \phi \cos \phi \leqslant(a+b) \sin \phi \cos \phi
$$

In the reflected system they are

$$
-\left(a^{\prime}+b^{\prime}\right) \sin \phi \cos \phi \leqslant\left(a^{\prime}-b^{\prime}\right) \sin \phi \cos \phi \leqslant\left(a^{\prime}+b^{\prime}\right) \sin \phi \cos \phi
$$

Both expressions guarantee the same thing; namely

$$
\begin{array}{ll}
0 \leqslant b ; & 0 \leqslant a \\
0 \leqslant a^{\prime} ; & 0 \leqslant b^{\prime}
\end{array}
$$


Since $a$ and $b$ are physically undetermined it is not of consequence whether the consecutive inequality first treats $a$ and then $b$ or vice versa. So, although the invariance of $M$ for reflexion is mathematically incomplete, it is physically speaking ensured.

Extension of these considerations to curvilinear and three-dimensional expressions requires a more elaborate treatment than can be given here.

\title{
REFERENCE
}

Bickley, W. G. \& Gibson, R. E. (1962). Via vector to tensor. London: English Universities Press.

\section{Estimation of the dynamic surface modulus of a generalized Gibson soil from the rocking frequency of rectangular foundations}

\author{
AWOJOBI, A. O. (1973). Géotechnique 23, No. 1, 23-31.
}

\section{Alpan, Israel Institute of Technology}

From the Author's approximate cquation (46) and the definition of the inertia ratio (p. 28) the natural frequency is obtained as

$$
\Omega^{2} \simeq\left(\pi b^{2} G_{0}-W h\right) / J
$$

Consideration of a simple one-degree-of-freedom model, using the appropriate subgrade modulus of an ideal half-space, furnishes the following relation (Alpan, 1963)

$$
\Omega^{2}=\left(\frac{\Delta M}{\theta}-W h\right) / J
$$

where the subgrade restoring moment per unit rotation

$$
\frac{\Delta M}{\theta}=\frac{E_{0}}{1-\nu_{2}} \cdot \frac{\pi B^{2} L}{16}
$$

In the Paper, the width $B=2 b$, the length $L=1$, and Poisson's ratio $=0 \cdot 5$ (incompressible soil). Equations (1) and (2) are thus seen to be identical.

Lack of sophistication is not necessarily a reason for rejecting an otherwise useful model.

\section{REFERENCE}

Alpan, I. (1963). Baugrund und Maschinenfundierung. Der Bauingenieur 38, No. 12, 459-462.

\section{Author's reply to I. AJpan}

Alpan's comment is a uscful addition to my references on the Paper. There is no intention on my part to reject a useful model on the grounds of lack of sophistication. The main point of the Paper is to justify the validity of simple models based on a homogeneous elastic half-space when they are applied to simulate actual soils whose moduli naturally increase with depth. This justification is necessary to ensure that further deductions based on the simple models can be regarded as reliable.

Furthermore, there is no other means of assessing the error involved in a simple model than by developing an exact formulation of the sophisticated model which represents a better approximation of the actual system. 